\title{
Analysing the diversification of cultural variants using longitudinal richness data
}

\author{
By ERIK GJesfJeld ${ }^{1}$, EnRICo R. CRema ${ }^{1}$, And AnNe KANDleR ${ }^{2}$ \\ University of Cambridge, Department of Archaeology, Cambridge, UK ${ }^{1}$, Max \\ Planck Institute for Evolutionary Anthropology, Department of Human Behavior, \\ Ecology and Culture, Leipzig, Germany ${ }^{2}$
}

\begin{abstract}
One of the most significant challenges for cultural evolution is the inference of macroevolutionary patterns from historical and archaeological sources of cultural data. Here, we examine the utility of diversification rate analysis for observing trends in the mode and tempo of cultural evolution using simulated cultural data sets. We explore a range of scenarios in which transmission modes, population size, and innovation rates change over time and generate population-frequency data. From this data, we extract longitudinal richness and further reduce its completeness through time-averaging and random sampling. Given that perfect population-level frequencies can rarely be assumed or even approximated from historical data, these simulated scenarios provide the grounds for exploring the inferential power of longitudinal richness data. Results suggest that diversification rate analysis can identify profiles of underlying changes in population size, innovation rates, and cultural transmission. Furthermore, our results highlight a series of methodological outcomes that can be used to enhance future research into the dynamic patterns of cultural evolution.

Keywords: Cultural Evolution, Diversification Rate Analysis, Archaeology, Richness, Cultural Diversity
\end{abstract}




\section{Introduction}

The archaeological record presents an interesting challenge for the field of cultural evolution. It is an often sparse and incomplete account of population-level patterns aggregated across spatial and temporal scales. These deficiencies lead to substantial problems for estimating long-term changes in cultural diversity and how these changes relate to various cultural, environmental, and demographic processes [e.g. $39,8,21,9,40,35,24,22,12,25]$. Nevertheless, the archaeological record is often the only direct empirical evidence of past cultural traditions and the forces affecting them.

The discrepancy between the quality of the available data and the ideal data to answer evolutionary research questions is not unique to archaeology. Darwin discussed at length the significance of fossil succession and the importance of palaeontology in identifying temporal changes in organisms [6]. Yet he was also greatly concerned with the incompleteness of the fossil record and that its many imperfections and inconsistencies could be used to argue against evolutionary theory [36, p.9]. These longstanding concerns have strongly influenced the research objectives of palaeontology, and later paleobiology. One of the most significant impacts is a commitment to rigorous, analytical approaches to quantifying taxonomic changes through time using evolutionary rates inferred from taxonomic richness and temporal information [36, 43]. As Alroy [1] acknowledges, measuring diversity in paleobiology cannot rely on knowing the distribution of individuals within a sample in the way that ecology and other disciplines measure diversity. Rather, the incompleteness of the fossil record makes it necessary to replace frequencies of individuals with taxonomic occurrences within time intervals [1].

In this paper, we draw inspiration from developments in paleobiology to explore how much information about the cultural evolutionary process can be extracted from measures of taxonomic richness combined with temporal information. We refer to this type of data as longitudinal richness data, which minimally contains an age estimate for the first and last appearance, and any occurrence in between, of each cultural variant (i.e. pottery ware, tool type, language, cultural practice, etc.). In other words, we propose to analyse time series data about the presence or absence of particular cultural variants with the aim to learn "something" about the underlying evolutionary forces. In general, this endeavour is supported by recent theoretical studies which show that knowledge of even a crude measure of the speed of cultural change provides more information about underlying cultural transmission processes than the exact knowledge of the frequency distribution at a single point in time [e.g. $16]$.

To date, approaches to uncover the evolutionary forces shaping the archaeological 
record have been mainly concerned with the reconstruction of different modes of cultural transmission, in particular the application of the neutral model for analysing the composition of artefact assemblages [e.g. 23, 2, 19] or more recently the development of generative inference frameworks [e.g. 4, 20, 15, 7, 27, 5, 14]. Neutral theory has often been applied to archaeological data in order to identify the absence (or presence) of directional selective forces in the population (see [13] for review). As caution needs to be exercised on how to interpret the consistency (or inconsistency) between the data and neutral expectations, modelling frameworks have been developed that simultaneously evaluate the consistency of a number of cultural transmission hypotheses with the available data while also accounting for demographic and cultural properties of the system. Those generative inference frameworks almost exclusively focus on the evaluation of the consistency between the observed and theoretical temporal changes in the frequencies of different cultural variants (such as the different types of decorated pottery) and have provided insights into the demographic and cultural assumptions that are able to generate frequency data. However, in many circumstances precise frequency information is hard and time-consuming to obtain, limiting the widespread use of these methods in archaeological applications. Longitudinal richness data, on the other hand, may be quicker and easier to acquire from the archaeological record and perhaps even more reliable in certain contexts. For example, frequency-level artefact data that has been aggregated across regions are likely to be strongly impacted by sampling differences between sites and a general unevenness in the intensity of habitation and artefact production over time. Longitudinal richness data, which only tallies presence or absence in a time interval, reduces the influence of this unevenness.

To study the suitability of longitudinal richness data in answering cultural evolutionary questions we use an approach based on the estimation of diversification rates. Broadly, diversification rates refer to the rate at which new things emerge (origination) and the rate and which existing things are lost (extinction). They have long served as a valuable tool in paleobiology for understanding the mode and tempo of evolutionary patterns [ $43,33,37,45,26,38,10,34,29,30]$, and whether the changes in taxonomic diversity over time are due to intrinsic causes (emergence of a new feature) or extrinsic causes (a changing environment) or a combination of both [44, p. 6187]. When applied to cultural evolution, diversification rate analysis broadly aims to capture the dynamics of when cultural variants are "born" through innovation and when cultural variants "die" from no longer being used and forgotten [18]. However, knowing the values of origination and extinction rates over time may not per se allow for conclusions about the underlying evolutionary processes producing the observed longitudinal richness data. To explore the inferential limits of this approach we use simulated data (subjected to time-averaging and sampling error) and examine how the calculated origination and extinction profiles relate to changes in the demographic and cultural assumptions, in particular to 
changes in population size, innovation rates, and processes of cultural transmission. The results of this analysis will enable us to understand which conclusions about underlying evolutionary processes can be made from longitudinal richness data.

\section{Methods}

\section{(a) Simulation Model}

To generate longitudinal richness data under known demographic and cultural processes we adapt a Wright-Fisher framework. More precisely, we consider a population of varying size evolving in discrete, non-overlapping generations. At time-step $t$ we assume a population of $N_{t}$ individuals that carry one of the $k_{t}$ cultural variants present at this time-step. Here, one time-step is considered to constitute a full cycle of transmission events. To generate the population at $t+1$ we assume $N_{t+1}$ naive individuals will each choose one of the $k_{t}$ variants $i$ present at $t$ with probability

$$
p_{i, t+1}=\frac{\left(x_{i, t} / N_{t}\right)^{1-b_{t+1}}}{\sum_{j=1}^{k_{t}}\left(x_{j, t} / N_{t}\right)^{1-b_{t+1}}}\left(1-\mu_{t+1}\right)
$$

where $x_{i, t}$ describes the absolute frequency of variant $i$ in the population at timestep $t, \mu$ the probability that a new, previously not seen cultural variant is introduced into the system and $b$ the strength of the frequency-dependent bias in the population. Frequency-dependent transmission is defined as the disproportional preference for either low-frequency variants (negative frequency-dependent transmission, also referred to as anti-conformist bias) or high-frequency variants (positive frequency-dependent transmission, also referred to as conformist bias). For $b>0$ transmission favours low-frequency variants whereas for $b<0$ transmission favours high-frequency variants. For $b=0$ we obtain unbiased transmission where each variant is chosen proportional to its relative frequency in the population. All parameters $\mu, b, N$ can vary with time.

Using the initial values of model parameters $\left(\mu_{0}, b_{0}, N_{0}\right)$, we let the cultural system reach its equilibrium [5] by running the model for 500 time-steps and then allow each of the parameters to vary temporally according to some predefined patterns (see Fig. 1). Importantly, these different patterns of change are chosen in a way so that they result in similar cultural dynamics as expressed by changes in the number of cultural variants (richness) through time (see Fig. 2). 
Change in population size. Changes in population size are modelled as linear growth, logistic growth, and exponential growth over 50 time-steps (see Fig. 1). The starting population size $N_{0}$ is approximately 1000 and the final population size $N_{50}$ is around 3000 . The innovation rate $\mu_{0}$ is held constant at 0.01 and the strength of frequency-dependent transmission $b_{0}$ at 0 , i.e. we assume unbiased cultural transmission.

Change in innovation rate. We consider three different scenarios for changes in the innovation rate. The first consists of a linear growth in the innovation rate from $\mu_{0}=0.005$ to $\mu_{50}=0.02$ over 50 time-steps. The second represents incremental change in the innovation rate with an increase by 0.0375 occurring every 10 timesteps. The third scenario highlights rapid change, at time-step 25 , that facilitates an immediate and sustained increase in the innovation rate from 0.005 to 0.02 . The population size is held constant at $N_{0}=2000$ and the strength of frequencydependent transmission $b_{0}$ at zero, i.e. unbiased cultural transmission.

Change in the process of cultural transmission. Lastly we vary the strength $b$ of frequency-dependent transmission. Once again, this includes a gradual linear change from a conformist $b_{0}=-0.03$ to an anti-conformist bias $b_{50}=0.03$ over 50 time-steps. Further, we model an incremental change from every 10 time-steps $[1-10: b=-0.03,11-20: b=-0.01,21-30: b=0,31-40: b=0.01$, $41-50: b=0.03$ ] as well as rapid change from -0.03 to 0.03 at time-step 25 . The population size is held constant at $N_{0}=2000$ and the innovation rate $\mu_{0}$ at 0.01 .

As one of our main interests in this paper is to understand the utility of this analysis to infer cultural evolutionary dynamics from incomplete data, we further reduce the completeness of our data. While numerous sources of bias influence the integrity of the archaeological record, time-averaging and sampling error are two of the most common and influential [e.g. 28]. We emulate time-averaging by aggregating the frequencies of cultural variants across five abutting time-steps $(1-5 ; 6-10 ; 11-$ $15 ; \ldots ; 45-50)$. This processes reduces the number of time intervals from 50 to 10. In addition, we randomly remove $90 \%$ of the time-averaged cultural variants to emulate sampling error for incomplete data-sets with a sampling fraction of $10 \%$.

In summary, our framework tracks the temporal change in the frequencies of all cultural variants introduced into the cultural system system under the nine different scenarios of change and different assumptions about the quality of the data. Finally, we extract, from each simulation run, the longitudinal richness data by recording the time-steps at which each cultural variant was first and last observed. 
(b) Diversification Rate Analysis using LiteRate

Rates of diversification are broadly defined here as the combination of the rate at which a new variants emerge (origination rate) and existing variants are lost (extinction rate). Numerous ways to calculate origination and extinction rates have been developed, with the advantages and disadvantages widely discussed in palaeontology [10]. A majority of rate measures start with a count of the number of new variants introduced or existing variants lost within a time interval and normalise the value by some measure of diversity [10]. The goal of this normalisation is to estimate a per-capita rate of origination and extinction for each time interval. When the time intervals are discrete units, as in this study, the origination rate can be broadly interpreted as the ratio between the number of new variants in a time-step divided by the richness of variants. Conversely, the extinction rate can be considered as the number of variants lost divided by the number of existing variants.

Diversification rate analysis is performed on both the complete and incomplete pseudo-data created from our simulation models using a unsupervised, machine learning algorithm known as "LiteRate" [18]. The program LiteRate models diversification rates using a probabilistic framework based on a linear birth-death process. The birth-death process assumes that the emergence and loss of cultural variants is influenced by the pool of existing variants and occurs in continuous time with a given set of rates. A benefit of the probabilistic framework outlined here is a reduction in the variability or noise that is inherent when calculating empirical diversification rates, as described above. For example, if no variant emerges or is lost in a time interval, the empirical origination or extinction rate would be zero. Thus, if the data being used is sparse with many time-steps not registering either a birth or death event, the origination and extinction rates will vary considerably. As LiteRate models origination and extinction events as a birth-death process, time-steps without events are viewed as stochasticity and accounted for in the estimation of diversification rates.

One key feature LiteRate provides is relaxing the assumption of constant origination and extinction rates throughout all time-steps. This is accomplished by essentially concatenating generalised birth-death processes together based on two events, which are the times $t$ of cultural variant emergence and loss, and the waiting times between them. The likelihood for this adaptive, continuous time birth-death process is detailed by Silvestro et al. [41] and Koch et al. [18].

This feature of LiteRate allows for evaluating when the most likely and most significant changes in origination and extinction rates occurred, known as rate shifts. Broadly, the number, timings, and magnitude of rate shifts is computed as the frequency of sampling a rate shift based on Reversible Jump Markov Chain Monte

Article submitted to Royal Society 
Carlo (RJMCMC) posterior samples. Like most MCMCs, new parameter values are proposed and accepted probabilistically over thousands or millions of proposals until the algorithm settles on a high-likelihood set of rate shifts (see [18] and the online tutorial for additional information). The LiteRate RJMCMC specifically evaluates the number of times a shift in the origination or extinction rate exceeds expectations based on priors (see Koch et al [18] for additional details).

An additional feature of LiteRate, or diversification rate analysis more broadly, is that we do not need to specify the evolutionary relationships between cultural variants prior to analysis. In other words, we do not require the creation of a phylogenetic tree in order to estimate diversity dynamics. This feature is particularly relevant when using archaeological or material culture data where we know that new variants are related to previous variants, but we do not always know the exact structure of these relationships. A final feature is that origination rates and extinction rates are not assumed to be linked together through time. This means extinction rates can exceed origination rates and potentially indicate periods of significant loss in diversity or "tipping points". It is this dynamic relationship between the origination rate and the extinction rate that often provides the best insight into underlying evolutionary mechanisms, such as diversification rate changes associated with an adaptive radiation process [11] or the "red queen" hypothesis [12, 29, 47].

\section{(i) LiteRate Settings}

Diversification rate analysis using LiteRate proceeded by analysing 50 out of 500 randomly selected replicates from each of the nine scenarios of change discussed above. The number of MCMC generations was set at 10 million with samples taken every 1000th generation. Here, a single analysis of 50 replicates using 10 parallel cores [46] lasted approximately 17 hours, for a total analysis time of approximately 150 hours (see supplemental information for details). The output of each LiteRate analysis is a series of files that include logs of origination and extinction rates and the RJMCMC generations. The performance of each RJMCMC analysis was evaluated by inspecting trace plots and the effective sample size (ESS), as calculated by the program Tracer [32]. After inspection, all 50 replicates for each model of cultural change were combined into a single log file, using the default function in LiteRate. Rate-through-time plots of origination and extinction rates were created based on the combined data using the R statistical environment [31]. All code and data used in this analysis is outlined in the supplemental information and can be found in the associated GitHub and FigShare repositories. 
1. Develop Models of Change

(a) Scenario A

1 Linear growth in population

2 Linear increase in innovation rate

3 Linear shift away from frequency-bias

(b) Scenario B

1 Incremental growth in population

2 Incremental increase in innovation rate

3 Incremental shift away from frequency-bias

(c) Scenario C

1 Abrupt growth in population

2 Abrupt increase in innovation rate

3 Abrupt change away from frequency-bias

2. Create pseudo-data using neutral model for each model of change. Model parameters include

$N_{t}=$ population size

$\mu_{t}=$ innovation rate

$b_{t}=$ strength of bias

(a) Scenario $\mathrm{A}$

$$
\begin{aligned}
1 & N_{t}=1000, \ldots, 3000 \\
& \mu_{0}=0.01 \\
& b_{0}=0 \\
2 & N_{0}=2000 \\
& \mu_{t}=0.005, \ldots, 0.2 \\
& b_{0}=0 \\
3 & N_{0}=2000 \\
& \mu_{0}=0.01 \\
& b_{t}=-0.03, \ldots, 0.03
\end{aligned}
$$

(b) Scenario B

$$
\begin{aligned}
1 & N_{t}=1000, \ldots, 3000 \\
& \mu_{0}=0.01 \\
& b_{0}=0 \\
2 & N_{0}=2000 \\
& \mu_{t}=0.005,0.00875 \\
& 0.0125,0.01625,0.02 \\
& b_{0}=0 \\
3 & N_{0}=2000 \\
& \mu_{0}=0.01 \\
& b_{t}=-0.03,-0.01,0,0.01,0.03
\end{aligned}
$$

(c) Scenario $\mathrm{C}$

$$
\begin{aligned}
1 & N_{0}=1000, \ldots, 3000 \\
& \mu_{0}=0.01 \\
& b_{0}=0 \\
2 & N_{0}=2000 \\
& \mu_{t}=0.05,0.2 \\
& b_{0}=0 \\
3 & N_{0}=2000 \\
& \mu_{0}=0.01 \\
& b_{t}=-0.03,0.03
\end{aligned}
$$

(d) Reduce completeness of pseudo-data through timeaveraging and random sampling

3. Diversification rate analysis of complete and incomplete data using LiteRate

(a) Count number of variants in each time-step (from pseudo-data)

(b) Assign origination and extinction date for each variant

(c) Randomly select 50 replicates (from 500) for diversification rate analysis

(d) Use LiteRate to estimate rates of diversification and significant rate shifts 
Table 1. Summary of Workflow

\section{Results}

We apply the diversification rate analysis described in section $2 \mathrm{~b}$ to the complete and incomplete longitudinal richness data generated by the nine different scenarios of cultural change with the aim to understand whether changes in population size, innovation rate, and transmission process will generate distinguishable origination and extinction profiles. The analysis of the complete data will provide us with a baseline of how much we can at most expect to extract from richness data. Any reduction in the quality of the data will naturally result in worse inference results, but will allow us to understand the requirements on the data so that the results of the analysis are meaningful and worth interpreting.

\section{(a) Analysis of the complete data}

We start our analysis by comparing the cultural scenarios where we assume that each of the model parameters $(\mu, b$ and $N)$ grow linearly over time and produce a similar increase in the number of unique cultural variants (Fig. 2A). The results of the diversification rate analysis on these linear models using LiteRate are shown in Fig. 3 . This includes a rate-through-time plot that can be broadly interpreted as rates of origination and extinction per time-step (left column). For example, if the origination rate is 0.15 at $t=10$ and there are 20 existing variants, we would expect that $3=20 \cdot 0.15$ new variants would emerge in that time-step.

The shape of extinction and origination profiles mirror richness trends shown in Fig. 2A by highlighting continual growth in cultural variant richness over time. This is indicated by origination rates being greater than extinction rates across all timesteps and all models (see left column of Fig. 3). Linear population growth and linear increases in innovation rates (models A1 and A2) result into a stable increase in richness. This is expected as the number of cultural variants gained and lost in the linear model stays, on average, constant through time and the diversification rates reflect this consistency. In contrast, the linear change from conformist to anti-conformist transmission (model A3) results in a decrease in both the origination and extinction rate. Conformist transmission supports high-frequency variants and consequently promotes lower richness with only a handful cultural variants being present while the opposite is true for anti-conformist transmission [3]. This means that when increasing $b$ the probability of low-frequency variants, such as new innovations, going extinct decreases, which in turn causes the extinction rate to 
decrease and overall variant count to increase. The decrease in the origination rate is simply caused by the increasing variant count over time.

The right column in Fig. 3, indicates the time-steps with the highest potential for a significant change in either the origination or extinction rate (i.e. a rate shift). As discussed above, LiteRate relaxes the assumption of constant rates by allowing origination and extinction rates to vary through time. Periods of significant changes in rates are determined by the proportion of RJMCMC posterior samples in which a rate shift was estimated to occur in each time-step. To infer whether a specific time-step has strong support for a rate shift, posterior sampling frequencies were associated with standard log Bayes factors (logBF) [17]. Broadly speaking, Bayes Factors compare two alternative hypotheses, in our case the presence or absence of a rate shift in a time-step. Positive support for a rate shift $2 \cdot \operatorname{logBF}>=2$ is indicated by the lower dashed line and strong support $2 \cdot \operatorname{logBF}>=6$ is indicated by the upper dashed line in Figs. 3, 4, and 5 (see [41] for additional details). Given that linear change is constant through time, there is no strong support for specific time-steps having significant changes in either origination or extinction rates (see Fig 3).

Next, we compare the cultural scenarios generated where each of the model parameters $\mu, b$ are subject to small, periodic changes every ten time-steps and $N$ is modelled as slow exponential growth. As before Fig. 2B shows that in all three scenarios the increase in the variant count over time is similar. Fig. 4 suggests a similar dynamic to the linear change models above. Origination and extinction rates based on population change, modelled by exponential growth, broadly reflect the underlying model with final origination rates rising closer to $t=50$. The incremental innovation scenario also demonstrates a similar pattern to linear change, although the first increase in the innovation rate is more clearly identified (model B2). This is due to the system leaving its initial equilibrium state when experiencing an increase in the innovation rate at time-step $t=10$ (from $\mu_{0}=0.005$ to $\mu_{10}=0.00875$ ). This change away from the equilibrium state is detected by LiteRate with strong support for a rate shift in the origination rate (see $4 \mathrm{~B} 2$ ).

Lastly, we compare the cultural scenarios where each of the model parameters $\mu$, $b$, and $N$ were subjected to a more sudden change. Once again, Fig. $2 \mathrm{C}$ shows that in all three scenarios the increase in the variant count over time is very similar. However, these changes demonstrate more obvious differences between our various models of change (Fig. 5). The population model (model C1), based on rapid logistic growth from $t=20$ to $t=30$, generally reflects this increase with a rise in the origination and extinction rates during this interval. Innovation rate changes (model C2) from 0.05 to 0.2 at $t=25$ resulting in sharp increase in the number of innovations and therefore the origination rate at this time-step. In the subsequent time-steps the origination rate can be interpreted as declining due to 
an increasing variant count, until its new equilibrium value is reached. But under an unbiased transmission regime a higher number of innovations, and therefore increased variant count, means a higher number of variant extinctions per time-step as well. So contrary to the behaviour of the origination rate, the extinction rate can be considered as increasing until its new equilibrium is reached.

If the transmission process changes abruptly from conformity $(b=-0.03)$ to anticonformity $(b=0.03)$, we observe a more extreme response (model $C 3)$ in the origination and extinction rates compared to the ones generated in population (model $\mathrm{C} 1$ ) and innovation rate (model $\mathrm{C} 2$ ) but for the same reasons. The conformist situation is characterised by only a few variants being present and any innovation is likely to go extinct immediately while the anti-conformist situation is characterised by a higher variant count and a lower probability that innovations go extinct immediately. Consequently, the change from conformity to anti-conformity can be interpreted as declines in both the extinction rate (due to the lower number of low-frequency variants going extinct) and the origination rate (due to the higher variant count) until the new equilibrium values have been reached.

As expected, abrupt changes to model parameters (scenario C) produce the strongest support for time-steps in which rate-shifts are estimated to occur. This feature is perhaps most valuable to research questions that aim to identify changes in the tempo of diversification. This would be most relevant to examining questions about gradual or punctuated culture change as well possible "tipping points" in the archaeological record [12].

In summary, there is evidence that diversification rate profiles associated with changing conformity bias $(b)$ are distinguishable from rate profiles that are produced from changes in population size $(N)$ or innovation $(\mu)$, at least with perfect data. We view this as a helpful conclusion when exploring whether changes in richness are more likely due to demographic factors or changes in cultural transmission processes.

(b) Analysis of Incomplete Data

Results from the analysis of incomplete data show similarities with the analysis of the complete data presented above. As expected, the loss of information impacts our results in two ways. The first is greater uncertainty around our origination and extinction rate estimates (see shaded areas of Fig. 6), due to using only a $10 \%$ sample of the complete data. The second difference between the complete and incomplete results is a decline in the ability to identify abrupt changes. Specifically, changes that occur rapidly in innovation rates and the strength of frequency-dependent bias are obvious in the complete data, but only vaguely recognised in the incomplete data 
(see 6, C1 and C2). This is most likely due to the blurring effect of time-averaging, which hinders the identification of changes between temporal periods. Despite these issues, it is encouraging that the general trends in origination and extinction rates are still visible in the analysis of our incomplete data. Perhaps most importantly, the diversification rate profiles associated with frequency-dependent bias still demonstrate declining rates of origination and extinction rates in combination with an overall increase in richness (see supplemental information, Fig. S1).

\section{Discussion}

As discussed above, the goal of the research presented here is to examine the inferential power of diversification rate analysis for understanding the evolutionary processes that shaped the observed longitudinal richness data. Based on the results, four general outcomes can be highlighted.

- Diversification rate analysis provides an intuitive way to assess whether the cultural system considered is characterised by an equilibrium or nonequilibrium dynamic. Under the former, both origination and extinction rates are expected to be constant and equal over time, while non-equilibrium conditions will exhibit a range of temporal origination and extinction profiles (see Figs. 3-5). Most analytical approaches proposed in the cultural transmission literature are based on equilibrium assumptions [see 13, for a discussion]. More complex methods such as generative inference frameworks [e.g. 5] require reliable knowledge of the initial state of the system (e.g. richness, variant frequencies) to be incorporated into the model. Either solutions are rarely satisfactory when examining archaeological data-sets, which often cover a wide temporal span with poor chronological resolution, leading to challenges in identifying the more likely changes in key parameters such as population size $N$, innovation rate $\mu$, or transmission process.

- Further, the shape of the temporal origination and extinction profiles may be indicative of the underlying process driving the change in variant richness over time. Based on our simulation model, we have shown that the shape of the origination and extinction profiles are very different for situations that demonstrate similar changes in richness over time. In our case, the underlying process of cultural transmission (frequency-dependent bias), as opposed to changes in demographic characteristics in population size or innovation rate, is responsible for the most significant differences in rate profiles. This is not to say that we expect a unique mapping between shape and underlying process, as we have already shown that changes in population size are not distinguishable from changes in innovation rate in our analysis. Nevertheless, 
those preliminary results give hope that we can use (real-world) longitudinal richness data in combination with the proposed diversification rate analysis to study the evolutionary processes that gave rise to them. We expect that results from such an analysis will at least help narrow the pool of potential hypotheses. For example, if we observe a pattern of declining origination and extinction rates in combination with a rise in overall richness we can use a simulation framework and look for processes, such as changes in the process of cultural transmission from conformity to anti-conformity, that (theoretically) produce such a pattern. But we reiterate, given the multitude of potential underlying processes we will most likely "only" be able to show consistency between process and empirical patterns with such an analysis.

- Even when data quality is poor, diversification rates produced using LiteRate still provide robust estimates for diversity dynamics (see Fig. 6). This does not mean that LiteRate directly alleviates concerns over incomplete data, but it does attempt to minimise their influence. If data incompleteness is assumed to be the product of randomly missing cultural variants, then estimates of diversification rates, including those from LiteRate, are largely unaffected. This is because the removal of a cultural variant does not influence the accuracy of rate estimation [42]. It should be noted that if the observed temporal duration of a cultural variant is believed to be much shorter than its true value, additional modelling approaches are often necessary. Specifically, the program PyRate [12, 41, 42] provides a series of features that are able to quantify uncertainty around times of origination and extinction as well as account for specific, biases such as age-dependent extinction. While the focus in this paper was on the incompleteness of archaeological data, many modern sources of data also lack critical information for studying cultural evolutionary processes. Broadly speaking, there are very few circumstances where the ideal data for testing cultural transmission hypotheses is available as most research questions require multiple proxies and diverse lines of evidence (see Yeh et al. this volume).

- The continued development of a diversification rates approach in cultural evolution requires considering a range of factors that are currently beyond the scope of this paper. One of our simplifying assumptions is that only one model parameter $(N, \mu, b)$ is changing at a time when it is far more likely that these parameters would be consistently changing and responding to each other. In order to manage this additional complexity, we suggest developing further scenarios that systematically vary multiple parameters and are more reflective of real-world events such as population bottlenecks, mass migrations, fashion cycles, or technological revolutions. Furthermore, we only consider two forms of bias in the creation of incomplete data, namely time-averaging and random sampling. More complex taphonomic processes 
such as age-dependent extinction or non-random preservation bias are likely to impact the empirical archaeological record in diverse ways. These existing challenges are largely methodological in nature and future research will be aimed at additional tactical simulations that further identify the inferential boundaries of our approach and its utility for illuminating the dynamics of cultural diversity.

In summary, our findings highlight that incomplete archaeological data, such as longitudinal richness data, can provide a valuable source of evidence for the inference of cultural macroevolution. Our approach using diversification rate analysis in combination with simulated data provides a baseline for understanding how changes in population size, innovation rate, and cultural transmission processes connect to rates of cultural variant emergence and loss over time. While our approach is not able to directly link patterns of cultural diversification to specific individual-level mechanisms, we can partially constrain the suite of processes that might have produced the observed patterns of artefact richness. Overall, the broader contribution of this work is the application of robust and flexible quantitative methods that help illuminate patterns in the tempo of cultural evolution and help to refine our understanding of cultural diversification dynamics. 


\section{Data Accessibility}

All of the data, code, and instructions to fully reproduce this analysis are deposited on GitHub https://github.com/erikgjes/Diversification_Rates_of_ Richness_Data as well as on FigShare https://doi.org/10.6084/m9.figshare. c. 5139077 .

\section{Author's Contributions}

$E G, E C$, and $A K$ designed the study and wrote the paper. EC and AK were responsible for modifying simulation model code and EG was responsible for the data analysis and diversification rate analysis.

\section{Competing Interests}

The authors have no competing interests.

\section{Funding}

EG acknowledges the McDonald Institute for Archaeological Research and the Renfrew Fellowship.

\section{Acknowledgements}

We would like to thank the guest editors (Eva Boon, Lucas Molleman, Pieter van den Berg, Franjo Wiessling) of this special issue and for the organisation of the workshop "Foundations of Cultural Evolution" at the Lorentz Center, University of Leiden. In addition, we would also like to thank Daniele Silvestro and Bernard Koch for their help and support in the continued development of LiteRate and its application to cultural evolution. 


\section{References}

[1] Alroy, J. (2020). On four measures of taxonomic richness. Paleobiology, 46(2):158-175.

[2] Bentley, R. A., Hahn, M. W., and Shennan, S. J. (2004). Random drift and culture change. Procedings of The Royal Society B, 271(1547):1443 - 1450.

[3] Boyd, R. and Richerson, P. J. (1985). Culture and the Evolutionary Process. Chicago University Press, Chicago, IL.

[4] Crema, E. R., Edinborough, K., Kerig, T., and Shennan, S. J. (2014). An Approximate Bayesian Computation approach for inferring patterns of cultural evolutionary change. Journal of Archaeological Science, 50:160-170.

[5] Crema, E. R., Kandler, A., and Shennan, S. (2016). Revealing patterns of cultural transmission from frequency data: equilibrium and non-equilibrium assumptions. Scientific Reports, 6(1):39122.

[6] Darwin, C. (1964). On the origin of species: A facsimile of the first edition. Harvard University Press, Cambridge, MA.

[7] Edinborough, K., Shennan, S., Crema, E., and Kerig, T. (2015). An abc of lithic arrowheads: a case study from south-eastern france. In Brink, K., Hydén, S., Jennbert, K., Larsson, L., and Olausson, D., editors, Neolithic Diversities. Acta Archaeologica Lundensia, Series 80, volume 65, pages 213-223. Department of Archaeology and Ancient History, Lund University.

[8] Eerkens, J. W. and Lipo, C. P. (2005). Cultural transmission, copying errors, and the generation of variation in material culture and the archaeological record. Journal of Anthropological Archaeology, 24(4):316-334.

[9] Foley, R. A. and Lahr, M. M. (2011). The evolution of the diversity of cultures. Philosophical Transactions of the Royal Society B: Biological Sciences, 366(1567):1080-1089.

[10] Foote, M. (2000-12). Origination and extinction components of taxonomic diversity: general problems. Paleobiology, 26(sp4):74-102.

[11] Gjesfjeld, E., Chang, J., Silvestro, D., Kelty, C., and Alfaro, M. (2016-05-10). Competition and extinction explain the evolution of diversity in american automobiles. Palgrave Communications, 2(1):1-6.

[12] Gjesfjeld, E., Silvestro, D., Chang, J., Koch, B., Foster, J. G., and Alfaro, M. E. (2020). A quantitative workflow for modeling diversification in material culture. PLOS ONE, 15(2):e0227579. 
[13] Kandler, A. and Crema, E. R. (2019). Analysing Cultural Frequency Data: Neutral Theory and Beyond. In Prentiss, A. M., editor, Handbook of Evolutionary Research in Archaeology, pages 83-108. Springer International Publishing, Cham, Switzerland.

[14] Kandler, A. and Powell, A. (2018). Generative inference for cultural evolution. Philosophical Transactions of the Royal Society B: Biological Sciences, 373(1743):20170056.

[15] Kandler, A. and Shennan, S. (2015). A generative inference framework for analysing patterns of cultural change in sparse population data with evidence for fashion trends in LBK culture. Journal of The Royal Society Interface, 12(113):20150905.

[16] Kandler, A., Wilder, B., and Fortunato, L. (2017). Inferring individual-level processes from population-level patterns in cultural evolution. Open Science, 4(9):170949.

[17] Kass, R. E. and Raftery, A. E. (1995). Bayes factors. Journal of the american statistical association, 90(430):773-795.

[18] Koch, B., Silvestro, D., and Foster, J. G. (2020). The evolutionary dynamics of cultural change (as told through the birth and brutal, blackened death of metal music). SocArXiv. https://doi.org/10.31235/osf.io/659bt.

[19] Kohler, T. A., VanBuskirk, S., and Ruscavage-Barz, S. (2004). Vessels and villages: evidence for conformist transmission in early village aggregations on the Pajarito Plateau, New Mexico. Journal of Anthroplogical Archaeology, 23(1):100118.

[20] Kovacevic, M., Shennan, S., Vanhaeren, M., d'Errico, F., and Thomas, M. G. (2015). Simulating Geographical Variation in Material Culture: Were Early Modern Humans in Europe Ethnically Structured? In Mesoudi, A. and Aoki, K., editors, Learning Strategies and Cultural Evolution during the Palaeolithic, Replacement of Neanderthals by Modern Humans Series, pages 103-120. Springer Japan, Tokyo.

[21] Lake, M. W. and Venti, J. (2009). Quantitative analysis of macroevolutionary patterning in technological evolution: bicycle design from 1800 to 2000 . In Shennan, S., editor, Pattern and Process in Cultural Evolution, pages 147-162. University of California Press, Berkeley, CA.

[22] Nakamura, M., Wakano, J. Y., Aoki, K., and Kobayashi, Y. (2019). The popularity spectrum applied to a cross-cultural question. Theoretical Population Biology, 133(2020):104-116. 
[23] Neiman, F. D. (1995). Stylistic Variation in Evolutionary Perspective: Inferences from Decorative Diversity and Interassemblage Distance in Illinois Woodland Ceramic Assemblages. American Anitquity, 60(1):7-36.

[24] O'Brien, M. J. and Buchanan, B. (2017). Cultural learning and the clovis colonization of north america. Evolutionary Anthropology: Issues, News, and Reviews, 26(6):270-284.

[25] O'Brien, M. J. and Bentley, R. A. (2020). Learning strategies and population dynamics during the pleistocene colonization of north america. In Groucutt, H. S., editor, Culture History and Convergent Evolution: Can We Detect Populations in Prehistory?, Vertebrate Paleobiology and Paleoanthropology, pages 261-281. Springer International Publishing.

[26] Pease, C. M. (1992). On the declining extinction and origination rates of fossil taxa. Paleobiology, 18(1):89-92.

[27] Porčić, M. and Nikolić, M. (2016). The approximate bayesian computation approach to reconstructing population dynamics and size from settlement data: demography of the Mesolithic-Neolithic transition at Lepenski Vir. Archaeological and Anthropological Sciences, 8(1):169-186.

[28] Premo, L. S. (2014). Cultural Transmission and Diversity in Time-Averaged Assemblages. Current Anthropology, 55(1):105-114.

[29] Quental, T. B. and Marshall, C. R. (2010). Diversity dynamics: molecular phylogenies need the fossil record. Trends in Ecology \& Evolution, 25(8):434441.

[30] Quental, T. B. and Marshall, C. R. (2013-07-19). How the red queen drives terrestrial mammals to extinction. Science, 341(6143):290-292.

[31] R Core Team (2020). R: A Language and Environment for Statistical Computing. R Foundation for Statistical Computing, Vienna, Austria.

[32] Rambaut, A. and Drummond, A. (2007). Tracer. http://beast.bio.ed.ac.uk/tracer.

[33] Raup, D. M. (1972). Taxonomic diversity during the phanerozoic. Science, 177(4054):1065-1071.

[34] Ricklefs, R. E. (2007-11-01). Estimating diversification rates from phylogenetic information. Trends in Ecology \& Evolution, 22(11):601-610.

[35] Rorabaugh, A. N. (2014). Impacts of drift and population bottlenecks on the cultural transmission of a neutral continuous trait: an agent based model. Journal of Archaeological Science, 49(Sep):255-264. 
[36] Sepkoski, D. (2012). Rereading the Fossil Record: The Growth of Paleobiology as an Evolutionary Discipline. University of Chicago Press, Chicago.

[37] Sepkoski, J. J. (1978). A kinetic model of phanerozoic taxonomic diversity i. analysis of marine orders. Paleobiology, 4(3):223-251.

[38] Sepkoski, Jr, J. J. (1998). Rates of speciation in the fossil record. Philosophical Transactions of the Royal Society B: Biological Sciences, 353(1366):315-326.

[39] Shennan, S. (2001). Demography and Cultural Innovations: a Model and its Implications for the Emergence of Modern Human Culture. Cambridge Archaeological Journal, 11(1):5-16.

[40] Shennan, S. (2011). Descent with modification and the archaeological record. Philosophical Transactions of the Royal Society B: Biological Sciences, 366:1071079.

[41] Silvestro, D., Antonelli, A., Salamin, N., and Meyer, X. (2019). Improved estimation of macroevolutionary rates from fossil data using a Bayesian framework. Paleobiology, 45(4):546-570.

[42] Silvestro, D., Schnitzler, J., Liow, L. H., Antonelli, A., and Salamin, N. (2014). Bayesian estimation of speciation and extinction from incomplete fossil occurrence data. Systematic biology, 63(3):349-367.

[43] Simpson, G. G. (1944). Tempo and mode in evolution. Columbia University Press, New York.

[44] Stadler, T. (2011-04-12). Mammalian phylogeny reveals recent diversification rate shifts. Proceedings of the National Academy of Sciences, 108(15):61876192.

[45] Stanley, S. M. (1979). Macroevolution: pattern and process. WH Freeman and Company, San Francisco.

[46] Tange, O. (2011). Gnu parallel - the command-line power tool. ;login: The USENIX Magazine, 36(1):42-47.

[47] Van Valen, L. (1973). A new evolutionary law. Evolutionary Theory, 1(1):1-30. 

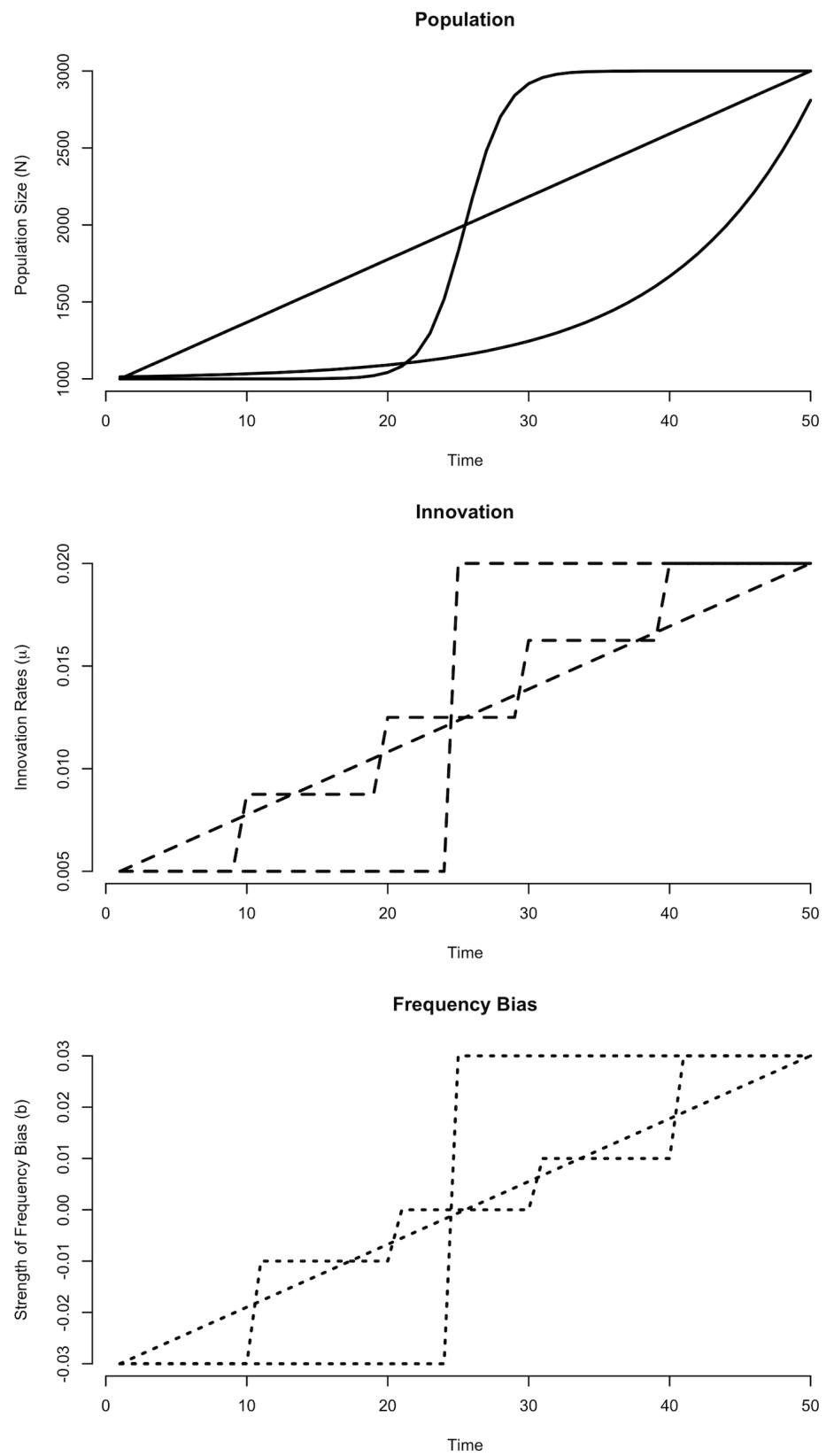

Figure 1. Visual representation of changes in model parameters under non-equilibrium scenarios. 

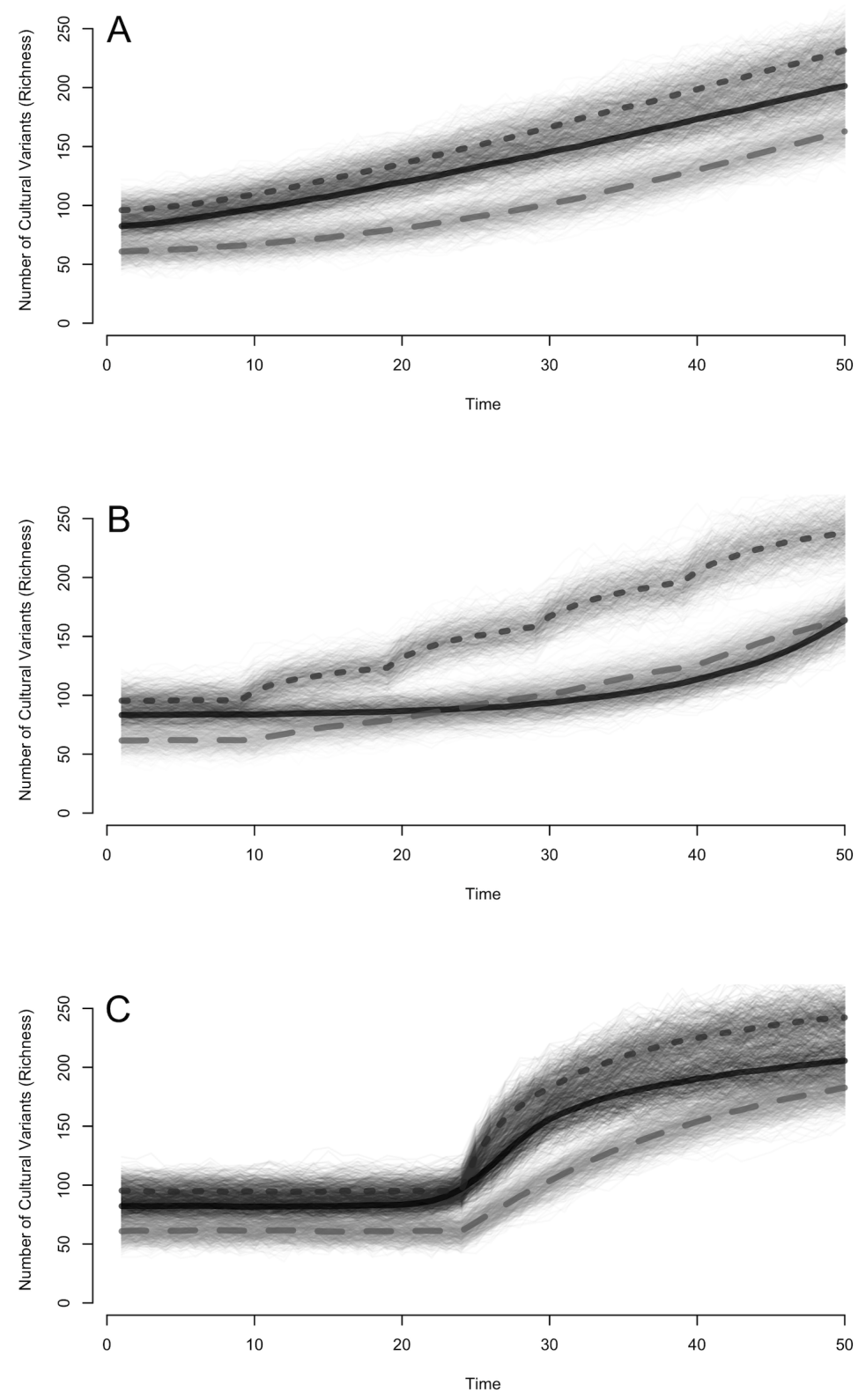

Figure 2. Counts of cultural variants for each model replicate across fifty time-steps. Scenario A represents linear change, scenario $B$ represents incremental change, and scenario $C$ represents abrupt change in model parameters. In each graph, the line indicates the mean richness of 500 replicates across changes in population size (solid line), innovation rate (short dashed), and frequency-bias (long dashed). 

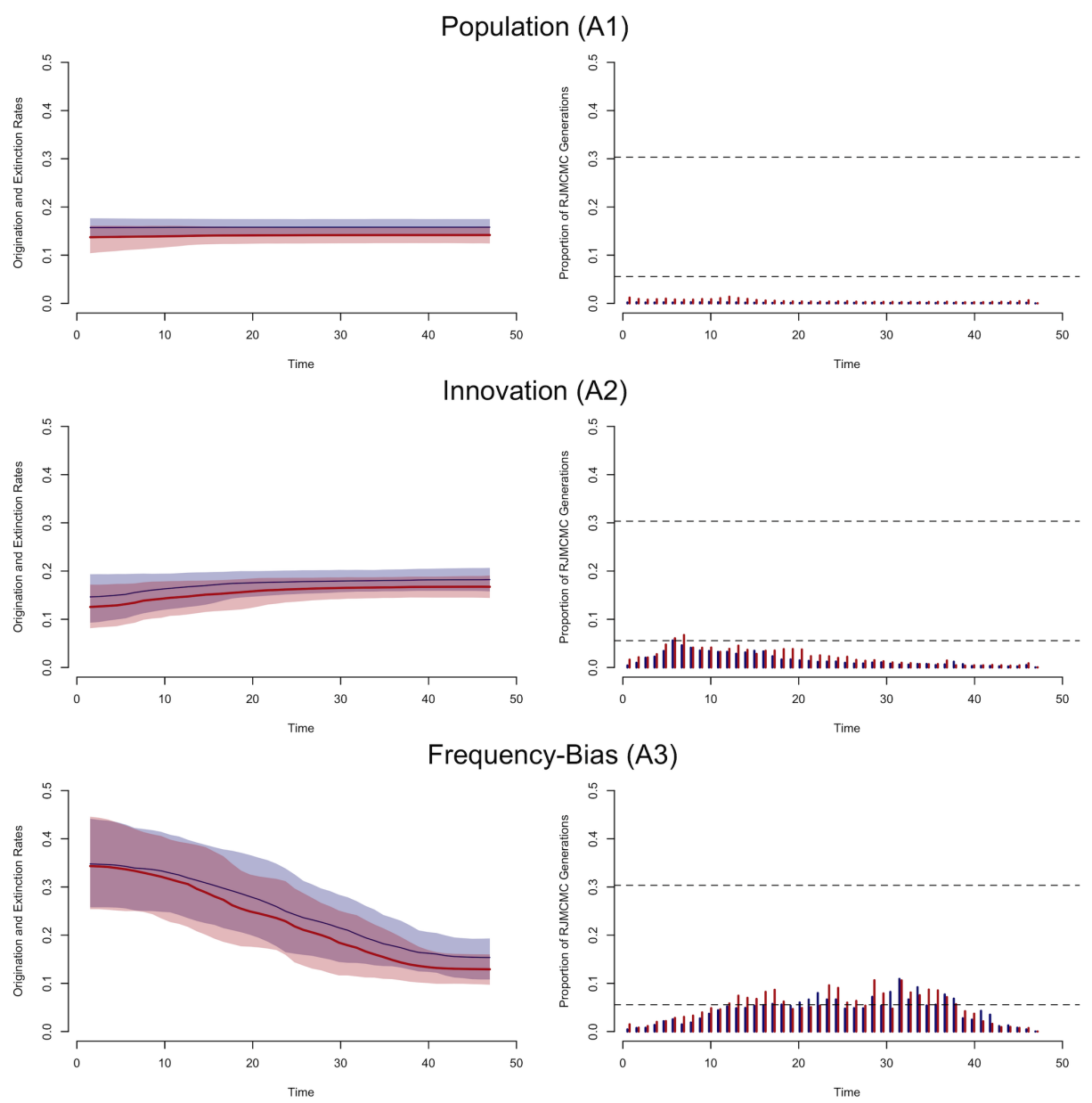

Figure 3. Results from Scenario A (linear change) with rows indicating different models of change. The left column highlights the estimated origination and extinction rates from LiteRate with $95 \%$ highest posterior density (shaded areas). The right column indicates the proportion of MCMC generations in which a rate shift was identified for each time-step in the posterior samples. Dashed lines correspond to Bayes Factor thresholds with the lower dashed line indicating positive support and the higher dashed line indicating strong support for the presence of a rate shift. 

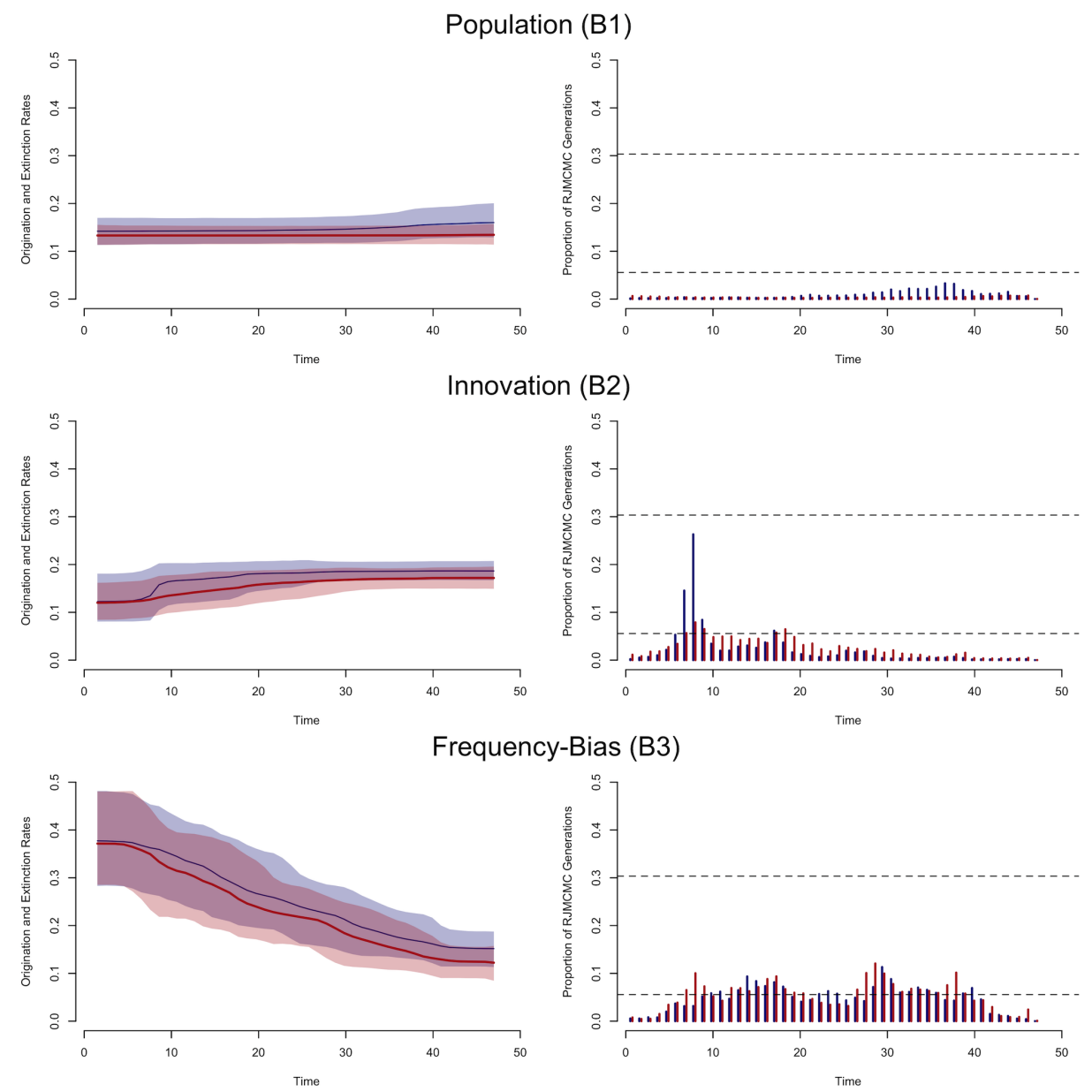

Figure 4. Results from Scenario B (incremental change) models. 

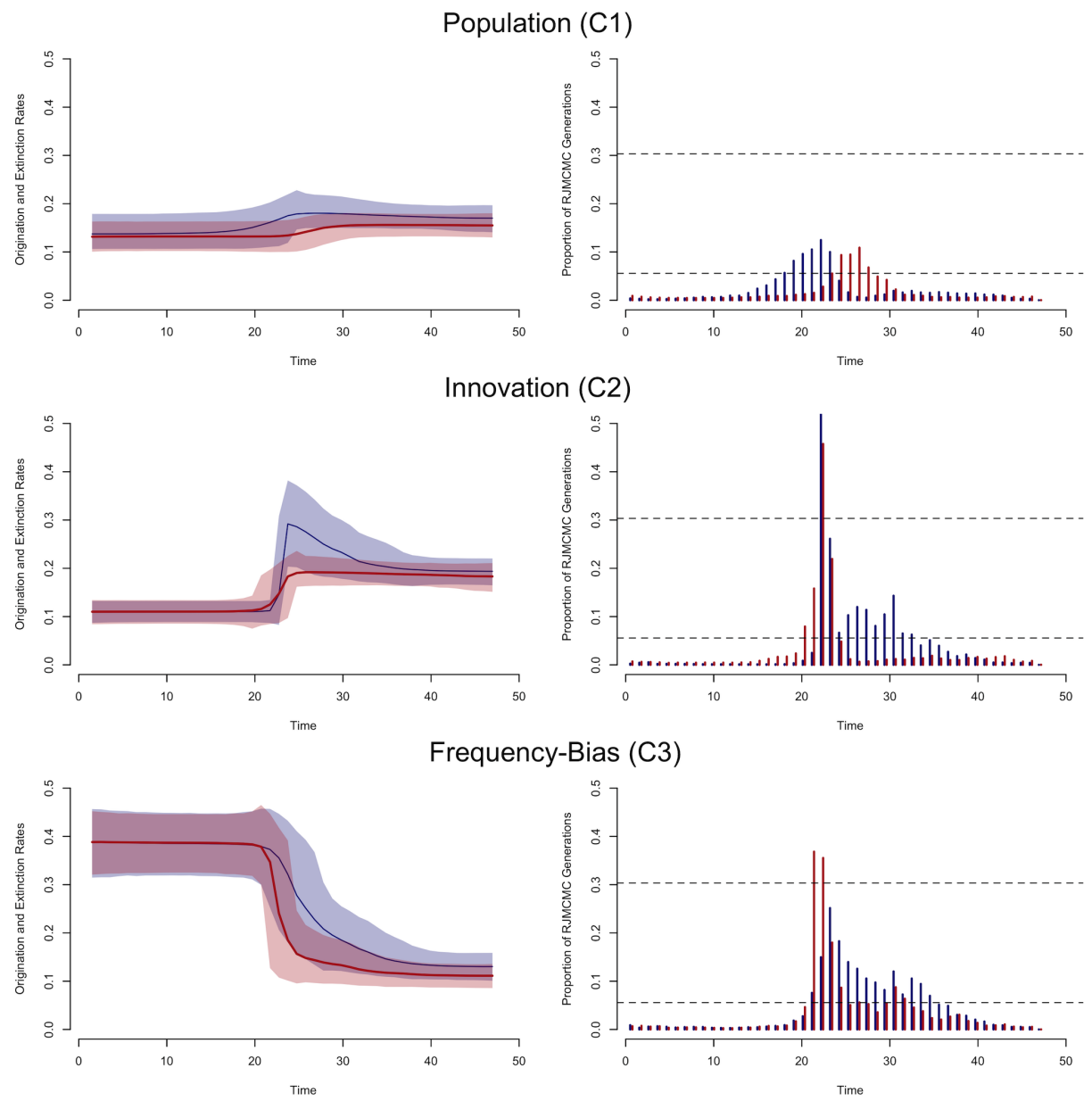

Figure 5. Results from Scenario C (abrupt change) models. 

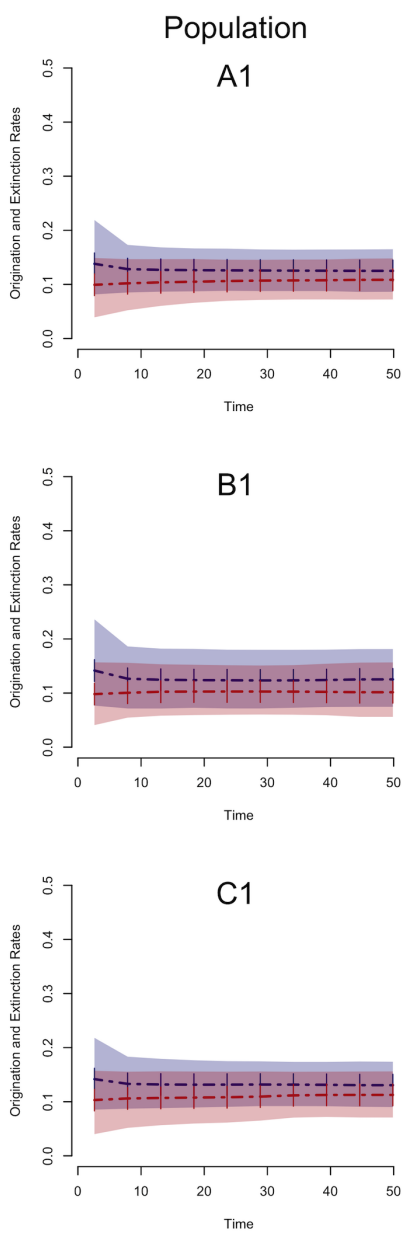

Innovation

A2

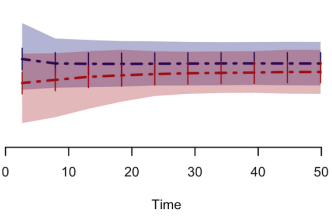

B2

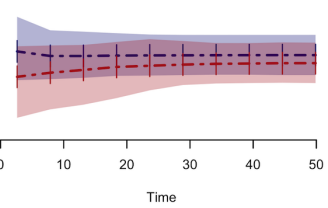

C2

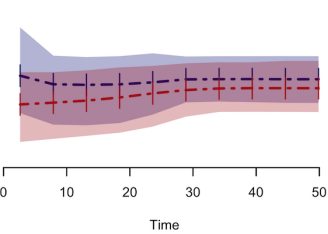

Frequency-Bias

A3

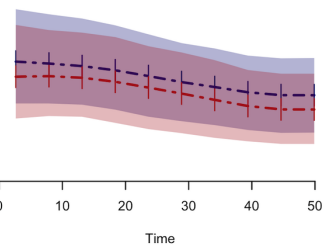

B3

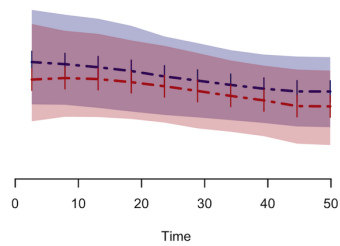

C3

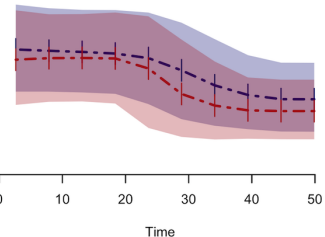

Figure 6. Origination and extinction rates from incomplete (dashed) cultural variant data along with 95\% HPD (shaded areas). Incomplete data has been time-averaged into 10 time-steps (vertical segments) with $90 \%$ of cultural variants randomly removed. 